\title{
Surface and Core Electronic Structure of Oxidized Silicon Nanocrystals
}

\author{
Noor A. Nama, ${ }^{1}$ Mudar A. Abdulsattar, ${ }^{2}$ and Ahmed M. Abdul-Lettif ${ }^{1}$ \\ ${ }^{1}$ Department of Physics, College of Science, University of Babylon, P.O. Box 4, Babylon, Iraq \\ ${ }^{2}$ Directorate of Materials Science, Ministry of Science and Technology, P.O. Box 8012, Baghdad, Iraq \\ Correspondence should be addressed to Mudar A. Abdulsattar, mudarahmed3@yahoo.com
}

Received 5 August 2010; Revised 12 September 2010; Accepted 9 December 2010

Academic Editor: Bohua Sun

Copyright ( 2010 Noor A. Nama et al. This is an open access article distributed under the Creative Commons Attribution License, which permits unrestricted use, distribution, and reproduction in any medium, provided the original work is properly cited.

\begin{abstract}
Ab initio restricted Hartree-Fock method within the framework of large unit cell formalism is used to simulate silicon nanocrystals between 216 and 1000 atoms (1.6-2.65 nm in diameter) that include Bravais and primitive cell multiples. The investigated properties include core and oxidized surface properties. Results revealed that electronic properties converge to some limit as the size of the nanocrystal increases. Increasing the size of the core of a nanocrystal resulted in an increase of the energy gap, valence band width, and cohesive energy. The lattice constant of the core and oxidized surface parts shows a decreasing trend as the nanocrystal increases in a size that converges to $5.28 \AA ̊$ in a good agreement with the experiment. Surface and core convergence to the same lattice constant reflects good adherence of oxide layer at the surface. The core density of states shows highly degenerate states that split at the oxygenated $(001)-(1 \times 1)$ surface due to symmetry breaking. The nanocrystal surface shows smaller gap and higher valence and conduction bands when compared to the core part, due to oxygen surface atoms and reduced structural symmetry. The smaller surface energy gap shows that energy gap of the nanocrystal is controlled by the surface part. Unlike the core part, the surface part shows a descending energy gap that proves its obedience to quantum confinement effects. Nanocrystal geometry proved to have some influence on all electronic properties including the energy gap.
\end{abstract}

\section{Introduction}

Silicon has many industrial uses and is considered one of the most important semiconductors [1]. It is the principal component of most semiconductor devices, most importantly integrated circuits. Silicon is widely used in the semiconductors industry because it remains a semiconductor at higher temperatures than the semiconductor germanium, and because its native oxide forms a better semiconductor/dielectric interface than any other material. The applications of silicon are in the electronic current conduction control (transistors), IC, detectors, solar cells and so forth. Nanocrystalline silicon (nc-Si) has small grains of crystalline silicon within the amorphous phase (a-Si). One of the most important advantages of nanocrystalline silicon is that it has increased stability over $(\mathrm{a}-\mathrm{Si})$.

Electronic structure of silicon has been studied extensively because it is widely used in electronic products [1-6]. On the other hand, the investigation of silicon nanocrystals is still an active field for investigation [3-6]. Silicon nanocrystals electronic structure of small hydrogenated or oxidized nanocrystals typically less than 500 atoms is performed using ab initio methods in the last years [3-6]. The present work addresses larger nanocrystals that have the size range of 2161000 silicon atoms. The present lower limit is chosen since it provides the first occurrence of core part (nearly diamond structure) that is far from surface reconstruction by more than one lattice constant. The present upper limit size has not been investigated before using self-consistent ab initio methods that requires longer execution computer times and higher memory which is the case of the present work.

Large unit cell method (LUC) coupled with ab initio Hartree-Fock self-consistent electronic structure calculations are used in the present work. LUC method was formulated and used before for several kinds of bulk materials including diamond structured materials [6-9]. Semiempirical LUC calculations were performed previously for silicon nanocrystals [10] with smaller number of atoms. This method uses 
$(\mathbf{k}=0)$ approximation, that is one point in the wave vector space. The LUC method is one kind of supercell methods with the above $\mathbf{k}=0$ restriction. In this method, and instead of adding more $\mathbf{k}$ points, the single central cell is expanded to contain more atoms, which are now called core atoms in the present work. We found this method very suitable for describing the core of a nanocrystal since it still has the outer dangling bonds that are saturated by surface atoms. The method does not expand to contain the whole space; instead it only contains the real number of atoms in a nanocrystal core that has nearly the exact diamond structure.

In most of the previous calculations of Si nanocrystals no clear distinction between core and surface properties of nanocrystals is made to the best of our knowledge. The distinction is important to understand the behavior of the different electronic properties and their relation to surface and core parts of the nanocrystal as we will see in present work. Another important reason for performing core and surface parts separately, is to reach a higher total number of atoms by summing the contributions of the different smaller parts of specific symmetry or size.

\section{Theory}

$\mathrm{Ab}$ initio self-consistent Hartree-Fock is used to obtain silicon nanocrystal molecular orbitals. Correlation corrections are neglected in the present calculations relying on Koopmans theorem [11]. This theorem states that comparisons of Hartree-Fock closed-shell results (which is the case in the present work) with experimental values suggest that in many cases the energetic corrections due to relaxation effects nearly cancel the corrections due to electron correlation. Simple STO-3G basis is used in the present work to be able to reach a higher number of core and surface atoms.

In the present work, we divided calculations into two parts, core and surface parts which is the traditional method used in microscopic-size solid-state calculations as shown in Figure 1. Normally surface effects do not penetrate more than four layers (one lattice constant) of the crystal surface [12]. On the other hand, short range $\mathrm{sp}^{3}$ bonds in diamond structured elements do not require more than fourth neighbor's interaction range to conduct electronic structure calculations successfully [8-10] using molecular orbital methods [13]. The upper two identical conditions will be applied in the present calculations. Previous silicon nanocrystals calculations $[3,5,14]$ showed that double bonding $(\mathrm{Si}=\mathrm{O})$ of oxygenated surfaces is preferred tosingle bridge bonding ( $\mathrm{Si}-\mathrm{O}-\mathrm{Si}$ ). Many reasons might support this bonding, such as surface curvature and larger lattice constants and bond lengths of nanocrystals. As a consequence of the above-mentioned reasons, the oxygen atoms are found in the vicinity of one silicon atom and far enough from other silicon surface atoms that promote double oxygen bonding.

Two kinds of core LUCs are investigated, namely cubic and parallelepiped cells. The cubic cells are multiples of diamond structure Bravais unit cells, while the parallelepiped cells are multiples of primitive diamond structure unit

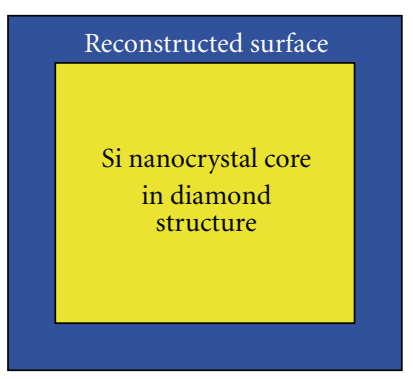

FIgure 1: (color online) A cross-section in Si cubic nanocrystal in which the outer area is the externally oxidized surface of one lattice constant width, while the inner area is the nanocrystal core and has nearly the exact diamond structure.

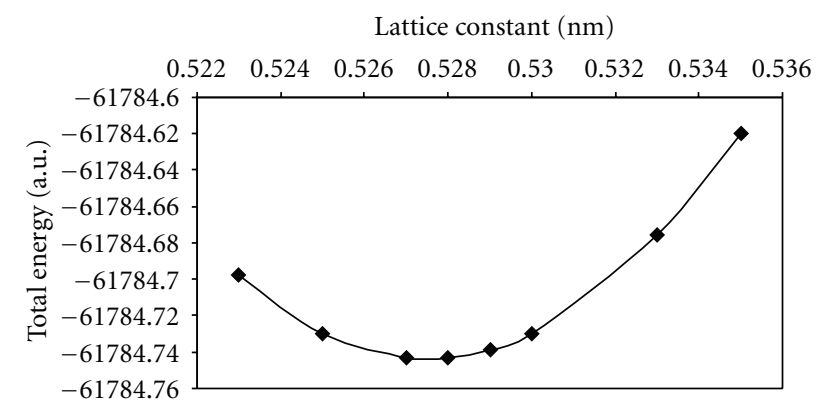

FIGURE 2: Total energy of $216 \mathrm{Si}$ atom nanocrystal core as a function of lattice constant.

cells [12]. Cubic core cells include 8, 64, and 216 atoms. Parallelepiped cells include 16, 54, 128, and 250 atoms.

\section{Calculations and Results}

We will perform the core part calculations using 3-D large unit cell method (LUC). The 2-D calculations for the oxygenated $(001)-(1 \times 1)$ surface is added to obtain a complete electronic structure view. The periodic boundary condition (PBC) method, available in GAUSSIAN 03 program [13] is used to perform the present tasks. Detailed formalism and applications of LUC can be found in the literature [6-10].

In Figure 2 total energy of $216 \mathrm{Si}$ atoms nanocrystal core as a function of lattice constant is plotted. This curve and similar curves for other LUCs are used to obtain equilibrium lattice constants for these cells. Figure 3 shows the equilibrium lattice constants obtained from the previous curves plotted against number of core atoms. Energy gap, valence band width, and cohesive energy are plotted against the number of core atoms in Figures $(4,5,6)$, respectively.

Three periodic slab stoichiometries were investigated to examine oxygenated $(001)-(1 \times 1)$ surface, namely, $\mathrm{Si}_{16} \mathrm{O}_{4}$, $\mathrm{Si}_{64} \mathrm{O}_{16}$, and $\mathrm{Si}_{144} \mathrm{O}_{36}$. These stoichiometries have surface areas $a^{2}, 4 a^{2}$, and $9 a^{2}$, respectively $((a)$ is the lattice constant). Figure 7 shows the surface energy gap of the oxygenated $(001)-(1 \times 1)$ surface as a function of surface area of Si nanocrystal facet. This figure is followed by a comparison between core and surface density of states in 


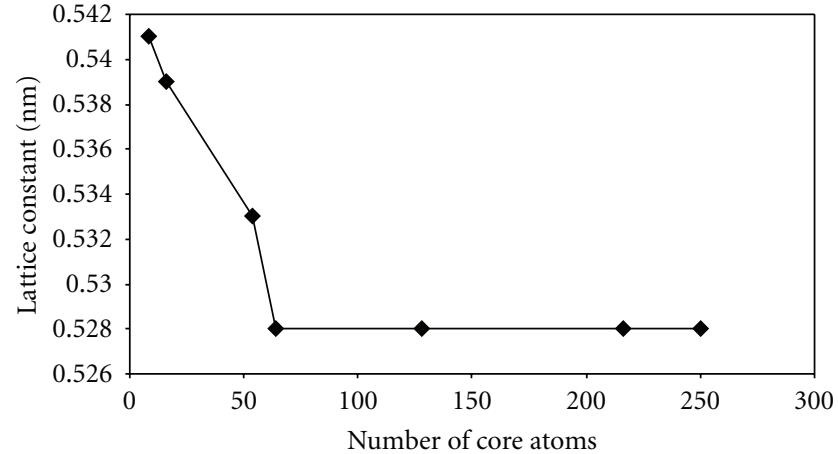

FIGURE 3: Lattice constant as a function of number of core atoms for Si nanocrystal.

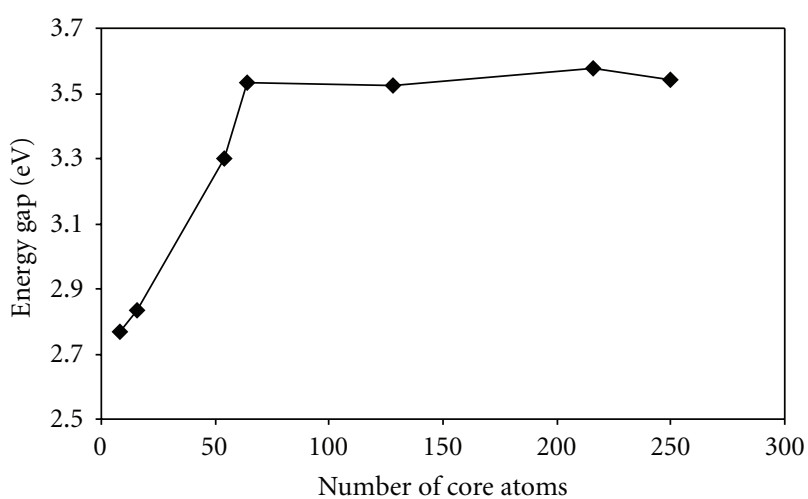

FIGURE 4: Energy gap of Si nanocrystal core as a function of number of atoms.

Figure 8. A Batman-shaped curve of ionic charges in an oxygenated $(001)-(1 \times 1)$ surface slab of periodic $\mathrm{Si}_{16} \mathrm{O}_{4}$ stoichiometry is shown in Figure 9.

\section{Discussion and Conclusions}

Because of symmetry, silicon nanocrystal core part has a unique single structure in one of its allotropies, namely, the diamond structure in the present work. This is the opposite case of surface multiple structures in which orientation, passivating atoms, and other situations in which surface structure changes accordingly. Figure 2 shows the variation of lattice constant with the number of core atoms. The variation of lattice constant is all what we need to assign the equilibrium geometry for the core part. The group of equilibrium constants for each of the investigated core sizes is ploted against the number of atoms in Figure 3. This figure shows that core lattice constant converges to some value as the nanocrystal grow up in size. This value is $5.28 \AA$ which is in a good agreement with the experimental value $5.43 \AA$ [1]. Surprisingly, the oxygenated $(001)-(1 \times 1)$ surface slab calculations of the nanocrystal converge to approximately the same lattice constant value of the core (5.28 $\AA$ ). This shows that negligible stresses are encountered at the interface

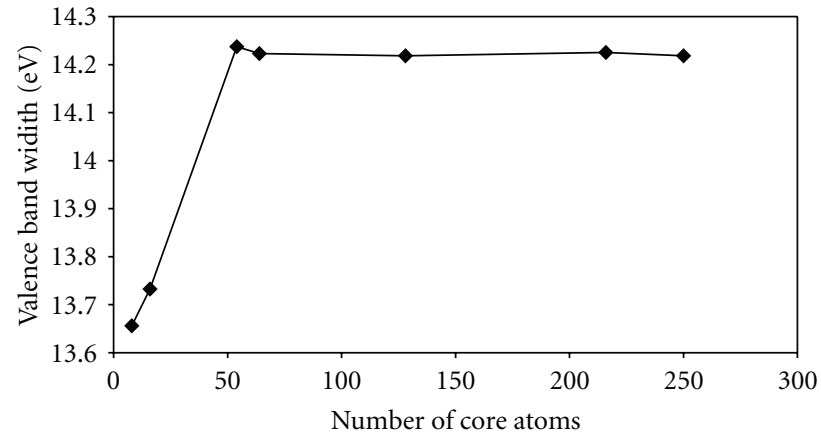

FIGURE 5: Valence band width of Si nanocrystals core as a function of number of atoms.

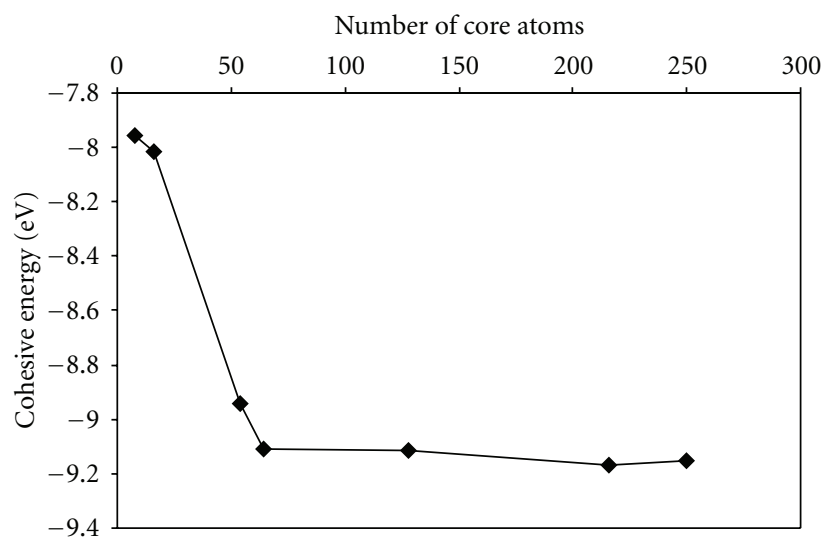

Figure 6: Cohesive energy for Si nanocrystal core as a function of number of atoms.

between surface and core parts, which eventually reflects good adherence of the oxide layer.

energy gap, valence band width and cohesive energy of the core part plotted against the number of core atoms in Figures 4-6, respectively, show a converging behavior as the number of core atoms increases. This convergence is associated with fluctuations depending on the geometry of the nanocrystal core. At the convergence plateau Bravais cubic lattices seem to have higher energy gaps, valence band width, and cohesive energy (absolute value). This is a clear indication of geometry effects on electronic structure of nanocrystals. Energy gap is inversely proportional to the surface area of core atoms (for a given size) as can be drawn from the fact that parallelepiped cells have less gap values than cubic ones. As an example for the geometry effect, we extracted the last four points in the energy gap Figure 4. These have the values $-3.53,-3.52,-3.57$ and $-3.54 \mathrm{eV}$, respectively. These points belong to $64,128,216$ and 250 core atoms, respectively. The 64 and 216 atoms are Bravais cells multiples (cubic) while 128 and 250 are primitive multiples (parallelepiped shape) [12]. We note from the above values that the first and third points (Bravais) are lower in energy than the surrounding second and fourth (primitive) points. These fluctuations appear more or less clearly (at least numerically) in other figures and 


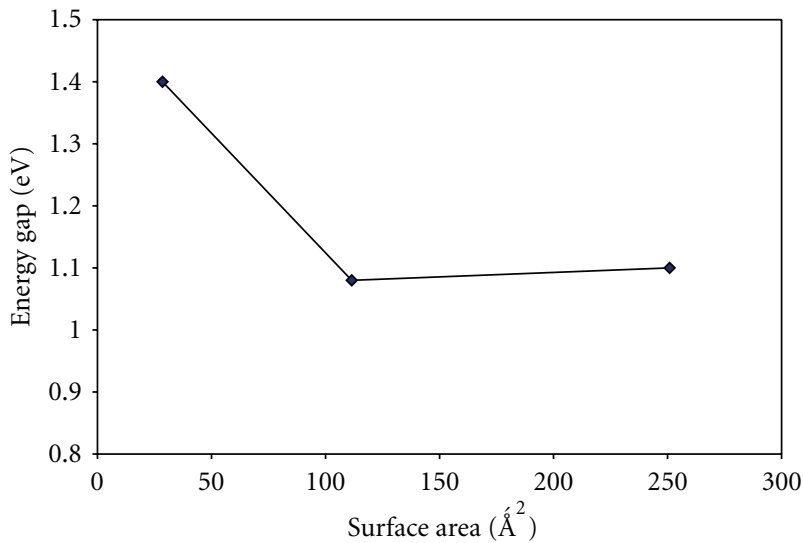

Figure 7: Energy gap of Si nanocrystal oxygenated $(001)-(1 \times 1)$ face as a function of surface area of nanocrystal facet.

indicate geometry effects on different electronic properties. This discrimination between the electronic properties is also applicable particularly to cohesive and valence band energies. These differences diminish as the nanocrystal grows up in size.

In Table 3 of [10], the semiempirical CNDO (complete neglect of differential overlap) method is used. This method is completely different from the present method since it uses semiempirical parameters to fit the data to experimental findings as in Table 1 of [10]. The present ab initio method does not use any parameters and are time consuming compared to the faster CNDO method that neglects a large number of the exact theory integrals. As a result of fitting, many of the fitted electronic properties are in a better comparison with the experiment, such as cohesive energy and energy gap.

Figure 7 shows the energy gap of oxygenated $(001)-(1 \times 1)$ silicon surface as a function of surface area of nanocrystal facet. From comparison of this figure with Figure 4, we can note that energy gap at the surface is much less than that at the core. As a consequence, the energy gap is controlled by the surface of nanocrystals. The descending energy gap shape of Figure 7 leads to the result that silicon nanocrystals at this size obey quantum confinement implications. Experimentally measured energy gap of silicon nanocrystals with a mean size of $4 \mathrm{~nm}$ embedded in $\mathrm{SiO}_{2}$ matrix is $1.7 \mathrm{eV}$ [15]. Although the size and matrix are different from the present size and interface, the present surface gap result $(1.1 \mathrm{eV})$ shows good agreement with the experiment. On the other hand, theoretical energy gap using the DFT (density function theory) method is reported as low as $1.6 \mathrm{eV}$ for oxygenated nanocrystals smaller than the present investigated nanocrystals [3]. This result is in excellent agreement with present work results, taking into account quantum confinement reduction of energy gap for larger sizes.

Hydrogenated silicon nanocrystals [16, 17] show wider energy gaps than oxygenated silicon nanocrystals. Surface modification by different atoms, has been discussed in [18]. The oxygen double bonding to the surface seems to be favorable in silicon nanocrystals interface and small nanocrystals $[19,20] . \mathrm{Si}=\mathrm{O}$ double bond is energetically optimized to 1.59 Á in the present work compared to $1.51 \AA$ in silica [21]. The energy gap sensitivity to surface modification is an indirect clue that surface conditions control the energy gap. The present works calculations are a direct proof of surface control of the energy gap.

The high value of the present core energy gap $(3.5 \mathrm{eV})$ is a trend of Hartree-Fock theory [9] which is opposed by a small gap (smaller than the experimentally observed values) in density function theory (DFT) [22]. We refer to [23] for a comparison of different models that evaluate the energy gap of related diamondoids structure. We can note that a high value is associated with Hartree-Fock results using Gaussian 98 program the predecessor of the presently used Gaussian 03 program. Another reason for the high value of core gap is that we are talking about nanocrystals of the range $1.6-2.65 \mathrm{~nm}$ in diameter that have a long way to follow to reach bulk properties. These nanocrystals tend to have large energy gap because silicon is an indirect gap semiconductor. For the smallest nanocrystals, only gamma points $(\Gamma)$ are present with only direct transitions (see [9, Table 1] and also references $[6,10]$ ). As nanocrystals grow in size, other symmetry points are added. The exact symmetry point that is needed for the lowest gap in silicon is created during size increase and might not be reached during the presently selected size range. It is interesting to note that direct silicon band gap is $3.4 \mathrm{eV}$ [9].

The present value of the cohesive energy and other properties can be greatly enhanced by merely changing the simple STO-3G basis used in the present calculations to a more sophisticated basis states. However, more sophisticated basis states consume more memory and computer time and will eventually prevent us from reaching a large number of atoms. As an example [24], the single zeta functions results differ from double zeta and multizeta functions results for silicon atoms by nearly $20 \mathrm{eV}$ (STO-3G functions used in the present work are comparable to single zeta functions).

Mesoscopic thermal conductance fluctuations of silicon nanowires are measured in [25]. The present fluctuations seem to be originating from a different geometrical origin, although fluctuations in conductance should also occur in the present case due to fluctuations in the energy gap [1]. The present results are related to size and geometry which are far from the sizes of [25] $\left(200 \times 100 \mathrm{~nm}^{2}\right.$ cross-section $)$.

Figure 8 shows a comparison between surface and bulk density of states. In this figure, we can note that degeneracy of states is reduced in surface case due to splitting of these states. Splitting occurs because of the cut-off of symmetry at the surface and the presence of oxygen atoms. As a consequence of this splitting, the energy gap is decreased and valence and conduction bands are increased.

Figure 9 shows the surface ionic (dielectric) charges of oxygenated $(001)-(1 \times 1)$ surface slab as a function of layer depth. The first and last atoms in the figure are for oxygen atoms which have negative charges due to their high affinity. Silicon atoms that are adjacent to oxygen atoms have positive charges. The second silicon neighbors to oxygen atoms have 


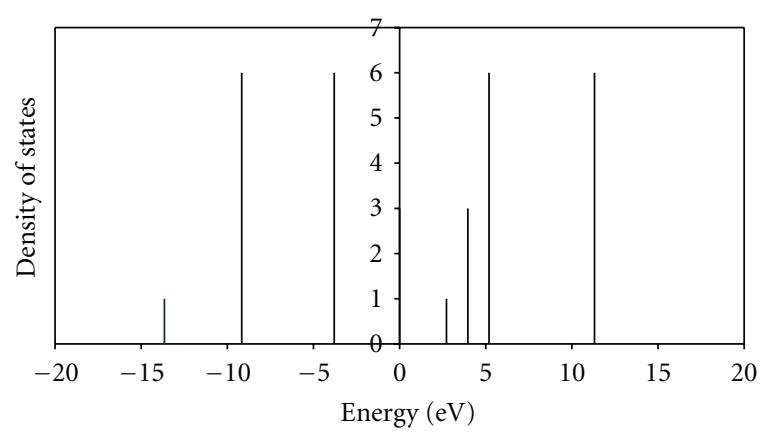

(a)

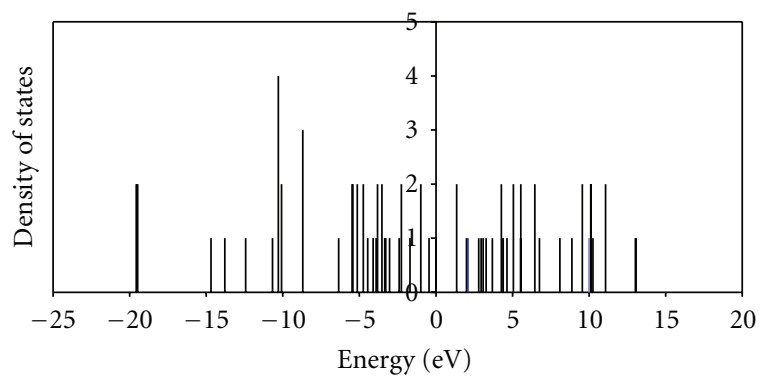

(b)

Figure 8: Degeneracy of states of 8 atoms LUC as a function of levels energy (a), and (b) surface density of states of oxygenated (001)-(1× 1) slab with $a^{2}$ surface area. Zero energy is assigned to the highest occupied molecular orbital (HOMO).

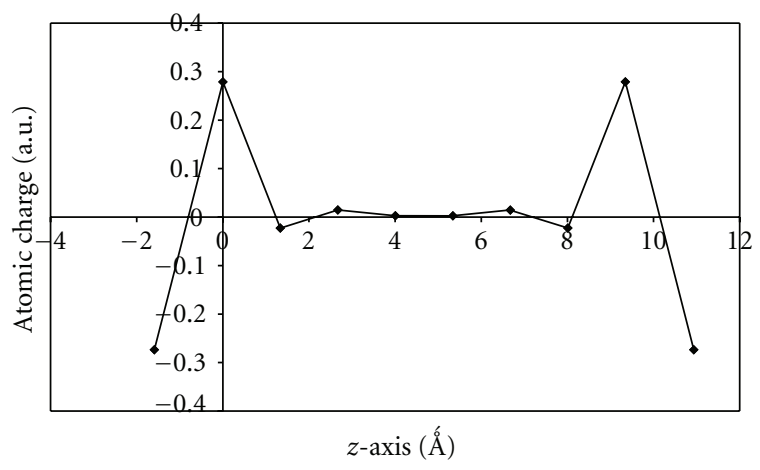

FIGURE 9: Ionic charges of oxygenated $(001)-(1 \times 1)$ surface slab as a function of layer depth.

negative charges while the third neighbors have positive charges. This damping oscillatory charges end at the fourth neighbors with practically zero charge. This curve is a good proof for the previously mentioned postulate of the adequacy of using four layers to represent the surface reconstruction depth.

Summarizing the above mentioned conclusions, the core part has a converging fluctuating energy gap, valence band width, and cohesive energy. These fluctuations are related to the geometry of the nanocrystal. The energy gap is controlled by the surface part of the nanocrystal with descending values that show its obedience to quantum confinement effects. The surface has damping oscillatory successive negative and positive layer charges. The surface part has a lower symmetry than the core part with smaller energy gap and wider valence and conduction bands. Surface and core parts have approximately the same lattice constant that reflects the good adherence of oxide layer at the surface.

\section{References}

[1] S. M. Sze and K. K. Ng, Physics of Semiconductor Devices, John Wiley \& Sons, New York, NY, USA, 3rd edition, 2007.

[2] S. J. Clark and G. J. Ackland, "Vibrational and elastic effects of point defects in silicon," Physical Review B, vol. 48, no. 15, pp. 10899-10908, 1993.

[3] M. Luppi and S. Ossicini, "Ab initio study on oxidized silicon clusters and silicon nanocrystals embedded in SiO: beyond the quantum confinement effect," Physical Review B, vol. 71, no. 3, Article ID 035340, pp. 1-15, 2005.

[4] T. Van Buuren, L. N. Dinh, L. L. Chase, W. J. Siekhaus, and L. J. Terminello, "Changes in the electronic properties of $\mathrm{Si}$ nanocrystals as a function of particle size," Physical Review Letters, vol. 80, no. 17, pp. 3803-3806, 1998.

[5] P. Carrier, "Curvature effects on optical response of $\mathrm{Si}$ nanocrystals in $\mathrm{SiO}_{2}$ having interface silicon suboxides," Physical Review B, vol. 80, no. 7, Article ID 075319, 2009.

[6] M. A. Abdulsattar and K. H. Al-Bayati, "Corrections and parametrization of semiempirical large unit cell method for covalent semiconductors," Physical Review B, vol. 75, no. 24, Article ID 245201, 2007.

[7] R. Evarestov, M. Petrashen, and E. Lodovskaya, "The translational symmetry in the molecular models of solids," Physica Status Solidi B, vol. 68, no. 1, pp. 453-461, 1975.

[8] A. H. Harker and F. P. Larkins, "A large unit cell semiempirical molecular orbital approach to the properties of solids. I. General theory," Journal of Physics C, vol. 12, no. 13, article no. 013, pp. 2487-2495, 1979.

[9] A. H. Harker and F. P. Larkins, "A large unit cell semiempirical molecular orbital approach to the properties of solids. II. Covalent materials: diamond and silicon," Journal of Physics C, vol. 12, no. 13, article 014, pp. 2497-2508, 1979.

[10] M. A. Abdulsattar, "Size effects of semiempirical large unit cell method in comparison with nanoclusters properties of diamond-structured covalent semiconductors," Physica E, vol. 41, no. 9, pp. 1679-1688, 2009.

[11] W. Hehre, L. Radom, P. Schileyer, and J. Pople, Ab Initio Molecular Orbital Theory, John Wiley \& Sons, New York, NY, USA, 1986.

[12] C. Kittel, Introduction to Solid State Physics, John Wiley \& Sons, New York, NY, USA, 5th edition, 1976.

[13] M. J. Frisch, G. W. Trucks, H. B. Schlegel et al., Gaussian 03, Revision B.01, Gaussian, Inc., Pittsburgh, Pa, USA, 2003.

[14] I. Vasiliev, J. R. Chelikowsky, and R. M. Martin, "Surface oxidation effects on the optical properties of silicon nanocrystals," Physical Review B, vol. 65, no. 12, Article ID 121302, pp. R1R4, 2002.

[15] L. Ding, T. P. Chen, Y. Liu, C. Y. Ng, and S. Fung, "Optical properties of silicon nanocrystals embedded in a SiO2 matrix," Physical Review B, vol. 72, no. 12, Article ID 125419, pp. 1-7, 2005.

[16] A. Puzder, A. J. Williamson, J. C. Grossman, and G. Galli, "Surface chemistry of silicon nanoclusters," Physical Review Letter, vol. 88, Article ID 097401, 2002.

[17] Z. Zhou, R. A. Friesner, and L. Brus, "Electronic structure of 1 to $2 \mathrm{~nm}$ diameter silicon core/shell nanocrystals: surface 
chemistry, optical spectra, charge transfer, and doping," Journal of the American Chemical Society, vol. 125, no. 50, pp. 15599-15607, 2003.

[18] A. Puzder, A. J. Williamson, J. C. Grossman, and G. Galli, "Surface chemistry of silicon nanoclusters," Physical Review Letters, vol. 88, no. 9, Article ID 097401, pp. 1-4, 2002.

[19] X. Chen, X. Pi, and D. Yang, "Bonding of oxygen at the oxide/nanocrystal interface of oxidized silicon nanocrystals: an ab initio study," Journal of Physical Chemistry C, vol. 114, no. 19 , pp. 8774-8781, 2010.

[20] R. J. Eyre, J. P. Goss, and P. R. Briddon, "Effect of progressive oxidation on the optical properties of small silicon quantum dots: a computational study," Physical Review B, vol. 77, no. 24, Article ID 245407, 2008.

[21] E. A. Robinson, "Characteristic vibrational frequencies of oxygen compounds of silicon, phosphorus, and chlorine: correlation of stretching frequencies and force constants with bond lengths and bond orders," Canadian Journal of Chemistry, vol. 41, pp. 3021-3033, 1963.

[22] J. Y. Raty, G. Galli, C. Bostedt, T. W. Van Buuren, and L. J. Terminello, "Quantum confinement and fullerenelike surface reconstructions in nanodiamonds," Physical Review Letters, vol. 90, no. 3, Article ID 037401, pp. 1-4, 2003.

[23] T. M. Willey, C. Bostedt, T. Van Buuren et al., "Observation of quantum confinement in the occupied states of diamond clusters," Physical Review B, vol. 74, no. 20, Article ID 205432, 2006.

[24] E. Clementi and C. Roetti, "Roothaan Hartree Fock atomic wavefunctions. Basis functions and their coefficients for ground and certain excited states of neutral and ionized atoms, $\mathrm{Z} \leq 54$," Atomic Data and Nuclear Data Tables, vol. 14, no. 3-4, pp. 177-478, 1974.

[25] J. S. Heron, T. Fournier, N. Mingo, and O. Bourgeois, "Mesoscopic size effects on the thermal conductance of silicon nanowire," Nano Letters, vol. 9, no. 5, pp. 1861-1865, 2009. 

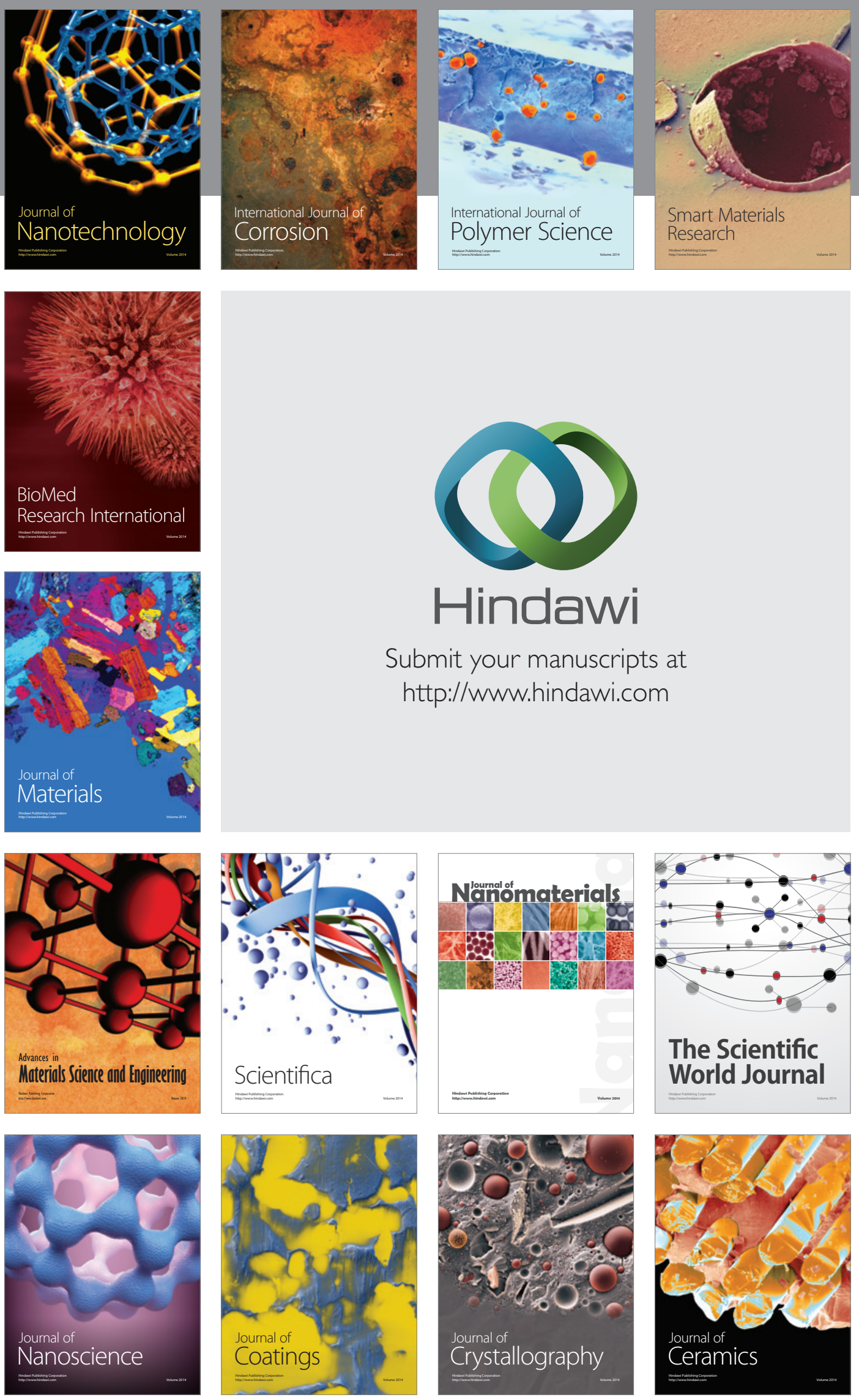

The Scientific World Journal

Submit your manuscripts at

http://www.hindawi.com

\section{World Journal}

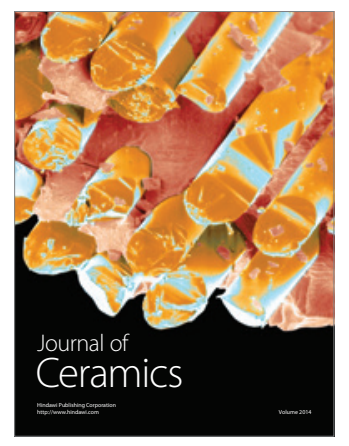

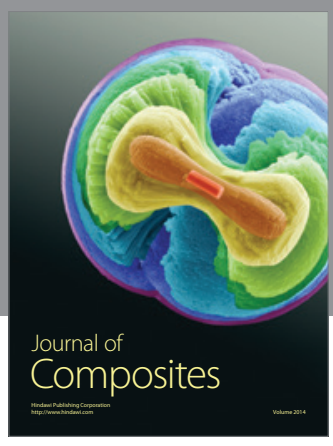
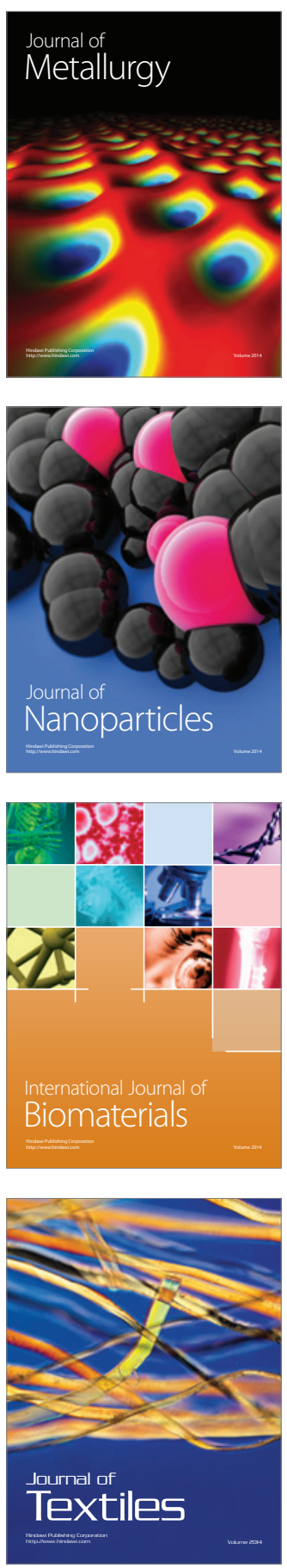